\title{
The N2K Consortium. III. Short\#Period Planets Orbiting HD 149143 and HD 109749
}

\section{Citation}

Fischer, Debra A., Gregory Laughlin, Geoffrey W. Marcy, R. Paul Butler, Steven S. Vogt, John A. Johnson, Gregory W. Henry, et al. 2006. "The N2K Consortium. III. Short\#Period Planets Orbiting HD 149143 and HD 109749." The Astrophysical Journal 637 (2): 1094-1101. https:// doi.org/10.1086/498557.

\section{Permanent link}

http://nrs.harvard.edu/urn-3:HUL.InstRepos:41397442

\section{Terms of Use}

This article was downloaded from Harvard University's DASH repository, and is made available under the terms and conditions applicable to Other Posted Material, as set forth at http:// nrs.harvard.edu/urn-3:HUL.InstRepos:dash.current.terms-of-use\#LAA

\section{Share Your Story}

The Harvard community has made this article openly available. Please share how this access benefits you. Submit a story.

Accessibility 


\title{
THE N2K CONSORTIUM. III. SHORT-PERIOD PLANETS ORBITING HD 149143 AND HD $109749^{1}$
}

\author{
Debra A. Fischer, ${ }^{2}$ Gregory Laughlin, ${ }^{3}$ Geoffrey W. Marcy, ${ }^{4}$ R. Paul Butler,${ }^{5}$ Steven S. Vogt, ${ }^{3}$ John A. Johnson, ${ }^{4}$ \\ Gregory W. Henry, ${ }^{6}$ Chris McCarthy, ${ }^{2}$ Mark Ammons, ${ }^{3}$ Sarah Robinson, ${ }^{3} J_{\text {ay }}$ Strader, ${ }^{3}$ Jeff A. Valenti, ${ }^{7}$ \\ P. R. McCullough, ${ }^{7}$ David Charbonneau, ${ }^{8}$ Joshua Haislip, ${ }^{9}$ Heather A. Knutson, ${ }^{8}$ Daniel E. Reichart, ${ }^{9}$ \\ Padric McGee, ${ }^{10}$ Berto Monard, ${ }^{11} \mathrm{~J}_{\text {ason T. Wright, }}{ }^{4}$ Shigeru Ida, ${ }^{12}$ Bun'ei Sato, ${ }^{13}$ and Dante Minniti ${ }^{14}$ \\ Received 2005 September 6; accepted 2005 October 3
}

\begin{abstract}
We report the detection of two short-period planets discovered at Keck Observatory. HD 149143 is a metal-rich G0 IV star with a planet of $M \sin i=1.33 M_{\mathrm{J}}$ and an orbital radius of 0.053 AU. The best-fit Keplerian model has an orbital period, $P=4.072$ days, semivelocity amplitude, $K=149.6 \mathrm{~m} \mathrm{~s}^{-1}$, and eccentricity, $e=0.016 \pm 0.01$. The host star is chromospherically inactive and metal-rich, with $[\mathrm{Fe} / \mathrm{H}]=0.26$. Based on the $T_{\text {eff }}$ and stellar luminosity, we derive a stellar radius of $1.49 R_{\odot}$. Photometric observations of HD 149143 were carried out using the automated photometric telescopes at Fairborn Observatory. HD 149143 is photometrically constant over the radial velocity period to $0.0003 \pm 0.0002 \mathrm{mag}$, supporting the existence of the planetary companion. No transits were detected down to a photometric limit of approximately $0.02 \%$, eliminating transiting planets with a variety of compositions and constraining the orbital inclination to less than $83^{\circ}$. A short-period planet was also detected around HD 109749, a G3 IV star. HD 109749 is chromospherically inactive, with $[\mathrm{Fe} / \mathrm{H}]=0.25$ and a stellar radius of 1.24. The radial velocities for HD 109749 are modeled by a Keplerian with $P=5.24$ days and $K=28.7 \mathrm{~m} \mathrm{~s}^{-1}$. The inferred planet mass is $M \sin i=0.28 M_{\mathrm{J}}$ and the semimajor axis of this orbit is 0.0635 AU. Photometry of HD 109749 was obtained with the SMARTS consortium telescope, the PROMPT telescope, and by transitsearch.org observers in Adelaide and Pretoria. These observations did not detect a decrement in the brightness of the host star at the predicted ephemeris time, and they constrain the orbital inclination to less than $85^{\circ}$ for gas giant planets with radii down to $0.7 R_{\mathrm{J}}$.
\end{abstract}

Subject headings: planetary systems — stars: individual (HD 149143, HD 109749)

On-line material: machine-readable tables

\section{INTRODUCTION}

The year 2005 marks the decadal anniversary of the detection of the first extrasolar giant planet, an $M \sin i=0.468 M_{\mathrm{J}}$ planet orbiting the star 51 Peg with a period of only 4.23 days (Mayor \& Queloz 1995). There are now more than 150 extrasolar planets known, and 29 of these have orbital periods shorter than 5 days. By virtue of proximity to their host stars, these so-called "hot Jupiters" have transit probabilities of about $10 \%$. Transits of bright stars provide information about the structure and atmosphere of extrasolar planets that currently is otherwise impos-

\footnotetext{
${ }^{1}$ Based on observations obtained at the W. M. Keck Observatory, which is operated as a scientific partnership among the California Institute of Technology, the University of California, and the National Aeronautics and Space Administration (NASA). The Observatory was made possible by the generous financial support of the W. M. Keck Foundation. The authors wish to recognize and acknowledge the very significant cultural role and reverence that the summit of Mauna Kea has always had within the indigenous Hawaiian community. We are most fortunate to have the opportunity to conduct observations from this mountain. Keck time has been granted by the National Optical Astronomy Observatory (NOAO) and NASA.

2 Department of Physics and Astronomy, San Francisco State University, San Francisco, CA 94132; fischer@stars.sfsu.edu.

3 University of California Observatories, University of California at Santa Cruz, Santa Cruz, CA 95064.

4 Department of Astronomy, University of California, Berkeley, CA 94720.

5 Department of Terrestrial Magnetism, Carnegie Institute of Washington DC, 5241 Broad Branch Road NW, Washington, DC, 20015-1305.

6 Center of Excellence in Information Systems, Tennessee State University, 330 10th Avenue North, Nashville, TN 37203; also Senior Research Associate, Department of Physics and Astronomy, Vanderbilt University, Nashville, TN 37235 .
}

sible to obtain. For example, the observation of a transit of HD 209458 (Charbonneau et al. 2000; Henry et al. 2000) revealed a bloated planet radius with neutral sodium detected in the dense lower atmosphere (Charbonneau et al. 2002) and an evaporating, trailing extended exosphere of hydrogen (Vidal-Madjar et al. 2004). The detection of a planet orbiting TrES-1 (Alonso et al. 2006) revealed a planet with radius similar to Jupiter; observation of the secondary eclipse for that planet placed limits on the Bond albedo (Charbonneau et al. 2005). The detection of a transiting planet around HD 149026 (Sato et al. 2005, hereafter Paper II) revealed a Saturn-mass planet with a radius of only $0.72 R_{\mathrm{J}}$. Theoretical models of the planetary interior suggest the presence of a substantial $\sim 70 M_{\oplus}$ core (Sato et al. 2005; Fortney et al. 2005). These transiting systems have provided a first glimpse at the interior structure of extrasolar planets and thereby

\footnotetext{
7 Space Telescope Science Institute, 3700 San Martin Drive, Baltimore, MD 21218 .

8 Harvard-Smithsonian Center for Astrophysics, 60 Garden Street, Cambridge, MA 02138.

9 Department of Physics and Astronomy, CB 3255 Phillips Hall, University of North Carolina, Chapel Hill, NC 27599.

${ }^{10}$ School of Chemistry and Physics, University of Adelaide, SA 5005, Australia.

11 Bronberg Observatory, Centurion 0157, Pretoria, South Africa.

12 Tokyo Institute of Technology, Ookayama, Meguro-ku, Tokyo 152-8551, Japan; and University of California Observatories, Lick Observatory, University of California, Santa Cruz, CA 95064.

13 Graduate School of Science and Technology, Kobe University, 1-1 Rokkodai, Nada, Kobe 657-8501, Japan.

${ }^{14}$ Department of Astronomy, Pontificia Universidad Catolica, Avenida Vicuña Mackenna 4860, Casilla 306 Santiago 200, Chile.
} 


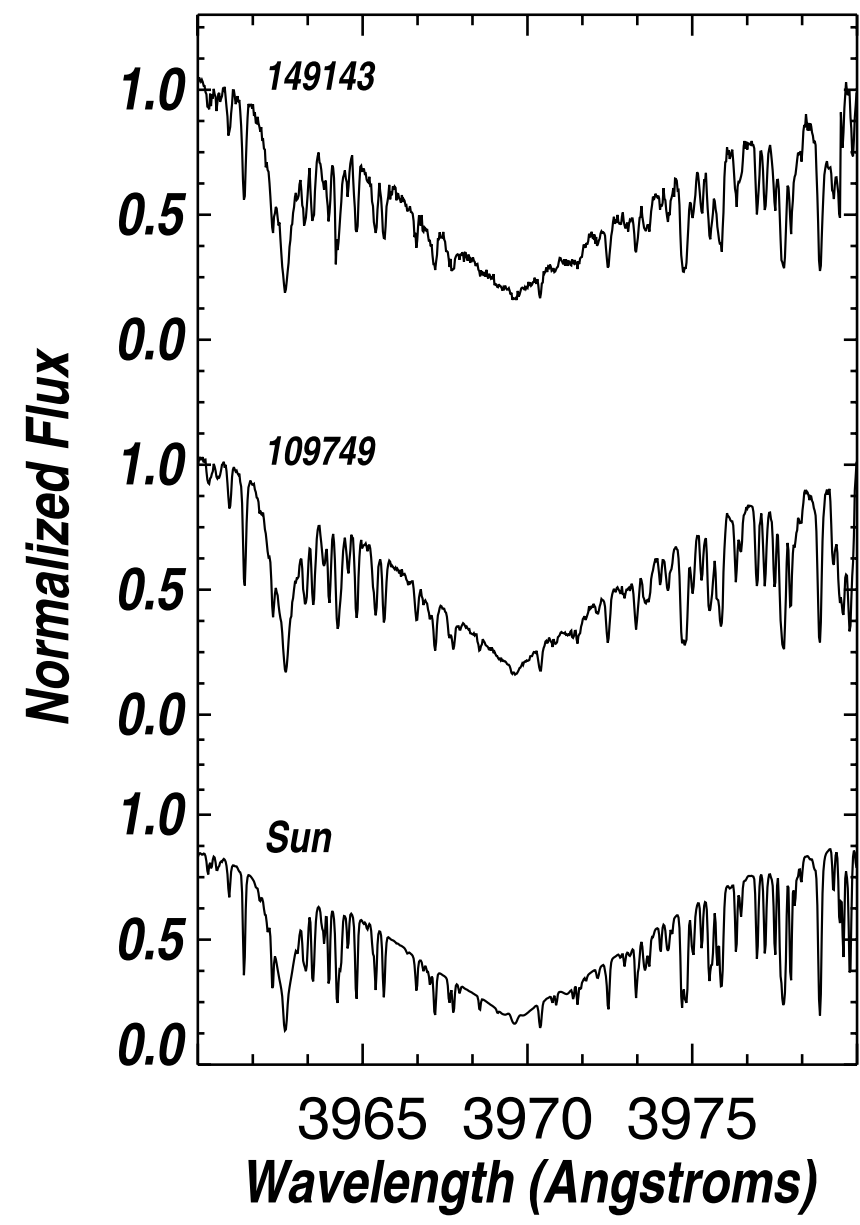

FIG. 1.- Ca H line for HD 149143 and HD 109749 plotted from top to bottom, with the $\mathrm{Ca} \mathrm{H}$ line of the Sun shown for comparison. No emission is seen in the line cores of either star, indicating low chromospheric activity.

provide boundary conditions for models of planetary atmospheres (Fortney et al. 2005; Burrows et al. 2005).

Nontransiting short-period planets also advance knowledge about planet formation and orbital evolution. At least $20 \%$ of stars with one planet show evidence for multiple planets (Marcy et al. 2005; Fischer et al. 2001). Short-period components of multiplanet systems can reside in resonance orbits or exhibit non-Keplerian interactions with sibling planets on timescales of just a few years (e.g., Laughlin \& Chambers 2001; Rivera \& Lissauer 2001), constraining scenarios for the orbital migration history of the system (Ford et al. 2005; Kley et al. 2005; Lee 2004; Lee \& Peale 2003). Modeling of measurable planet-planet interactions can establish the degree of coplanarity of the planetary orbits (e.g., Rivera et al. 2005) for some systems in which the radial velocity signal-to-noise ratio is sufficiently high.

The discovery of more hot Jupiters will shed light on current statistical anomalies observed in the distributions of extrasolar planets. For example, there is an apparent pile-up of planets with orbital periods between 3-10 days and a dramatic drop in both the number of planets with orbital periods shorter than 3 days and planets in intermediate orbits (10-100 days). While three out of four transiting planets from the OGLE survey (Udalski et al. 2002a 2002b, 2003; Konacki et al. 2003; Bouchy et al. 2004) have orbital periods of about 1.5 days, these "very hot Jupiters" have not yet been detected in Doppler surveys of nearby, bright stars, indicating that they are intrinsically very rare.

\section{N2K CONSORTIUM}

Short-period planets can be discovered quickly in high-precision radial velocity (RV) surveys. Virtually all short-period planets with $M \sin i>0.5 M_{\mathrm{J}}$ have been culled from ongoing Doppler programs. The N2K consortium (Fischer et al. 2005) was assembled to carry out a distributed Doppler survey of 2000 stars not on current Doppler programs. The sample is enhanced with metalrich stars to increase the detection probability (Fischer \& Valenti 2005). The initial metallicity estimate was made using the broadband (BVJHK) calibration of Ammons et al. (2006), and lowresolution spectroscopic metallicity measurements were obtained for more than 1000 stars (Robinson et al. 2006). As telescope time is allocated a set of three consecutive observations is obtained. Our Monte Carlo simulations show that an rms scatter between 15 and $80 \mathrm{~m} \mathrm{~s}^{-1}$ in these three RVs is a good diagnostic for the presence of a short-period planet. Follow-up Doppler observations and photometric observations to monitor the star for transits (described in Paper II) are initiated for all short-period planet candidates.

\section{HD 149143}

HD 149143 is a G0 IV star with $V=7.89$ and $B-V=0.68$. The Hipparcos parallax (ESA 1997) gives a distance of $63.5 \mathrm{pc}$ and an absolute visual magnitude, $M_{V}=3.87$. From the bolometric luminosity and our spectroscopic $T_{\text {eff }}$, we derive a stellar radius of $1.49 \pm 0.1 R_{\odot}$. Using the bolometric luminosity, $L=$ $2.39 L_{\odot}$ and our spectroscopic temperature and metallicity to interpolate over the Yale evolutionary tracks (DeMarque et al. 2004), we derive a stellar mass of $1.2 \pm 0.1 M_{\odot}$ and stellar age of $4 \pm 1.4 \mathrm{Gyr}$. The $\mathrm{Ca} \mathrm{H}$ and $\mathrm{K}$ lines, plotted in Figure 1, show that the star is chromospherically inactive. We measure $S_{\mathrm{HK}}=$ 0.167 and $\log R_{\mathrm{HK}}^{\prime}=-4.97$ and derive a rotational period of 28 days following the calibration of Noyes et al. (1984). The initial metallicity estimate for this star was $[\mathrm{Fe} / \mathrm{H}]=0.33 \pm 0.15$ (Ammons et al. 2006). Our medium-resolution calibrations (Robinson et al. 2006) provided further evidence of a high metallicity, yielding $[\mathrm{Fe} / \mathrm{H}]=0.30, T_{\text {eff }}=5896$, and $\log g=4.34$. Our high-resolution spectroscopic analysis (described in Valenti \& Fischer 2005) yields $[\mathrm{Fe} / \mathrm{H}]=0.26 \pm 0.05, T_{\text {eff }}=5884 \pm 50 \mathrm{~K}$, $\log g=4.071 \pm 0.07$, and $v \sin i=4.0 \pm 0.5 \mathrm{~km} \mathrm{~s}^{-1}$. The stellar parameters are summarized in Table 1.

\subsection{Doppler Observations of HD 149143}

Doppler observations were made at the Keck telescope using HIRES (Vogt et al. 1994) with an iodine cell to model instrumental

TABLE 1

Stellar Parameters

\begin{tabular}{|c|c|c|}
\hline Parameter & HD 149143 & HD109749 \\
\hline$V \ldots$ & 7.89 & 8.09 \\
\hline$\ldots \ldots \ldots$ & 3.87 & 4.22 \\
\hline$B-V$ & 0.680 & 0.714 \\
\hline 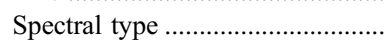 & G0 IV & G3 IV \\
\hline Distance $(\mathrm{pc})$ & 63.5 & 59 \\
\hline$[\mathrm{Fe} / \mathrm{H}]$ & $0.26(0.05)$ & $0.25(0.05)$ \\
\hline$T_{\mathrm{eff}}(\mathrm{K})$ & $5884(50)$ & $5903(50)$ \\
\hline$v \sin i\left(\mathrm{~km} \mathrm{~s}^{-1}\right) \ldots \ldots \ldots \ldots \ldots \ldots \ldots \ldots \ldots \ldots \ldots$ & $4.0(0.5)$ & $2.5(0.5)$ \\
\hline $\log g$ & $4.071(0.07)$ & $4.403(0.07)$ \\
\hline$M_{\mathrm{STAR}}\left(M_{\odot}\right)$ & $1.21(0.1)$ & $1.2(0.1)$ \\
\hline$R_{\mathrm{STAR}}\left(R_{\odot}\right)$ & $1.49(0.1)$ & $1.24(0.1)$ \\
\hline$S_{\mathrm{HK}}$ & 0.167 & 0.155 \\
\hline $\log R_{H K}^{\prime}$ & -4.97 & -5.04 \\
\hline$P_{\mathrm{ROT}}$ (days) & 28 & 34 \\
\hline
\end{tabular}


TABLE 2

Radial Velocities for HD 149143

\begin{tabular}{|c|c|c|}
\hline $\mathrm{JD}-2,440,000$ & $\begin{array}{c}\mathrm{RV} \\
\left(\mathrm{m} \mathrm{s}^{-1}\right)\end{array}$ & $\begin{array}{l}\text { Uncertainty } \\
\left(\mathrm{m} \mathrm{s}^{-1}\right)\end{array}$ \\
\hline 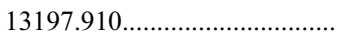 & 0.00 & 5.55 \\
\hline 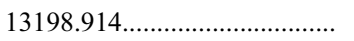 & -90.05 & 5.62 \\
\hline 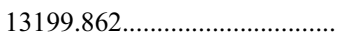 & 86.12 & 5.52 \\
\hline 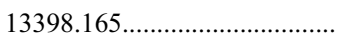 & -91.05 & 3.73 \\
\hline $13399.153 \ldots \ldots \ldots \ldots \ldots \ldots \ldots \ldots$ & 37.55 & 3.99 \\
\hline $13401.151 \ldots \ldots \ldots \ldots \ldots \ldots \ldots \ldots \ldots$ & 92.43 & 3.76 \\
\hline $13425.169 \ldots \ldots \ldots \ldots \ldots \ldots \ldots$ & 163.09 & 3.04 \\
\hline 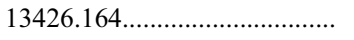 & -39.58 & 2.99 \\
\hline $13427.149 \ldots \ldots \ldots \ldots \ldots \ldots \ldots \ldots . .$. & -59.90 & 3.28 \\
\hline 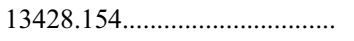 & 146.65 & 3.32 \\
\hline $13429.107 \ldots \ldots \ldots \ldots \ldots \ldots \ldots . .$. & 184.50 & 2.93 \\
\hline $13430.040 \ldots \ldots \ldots \ldots \ldots \ldots \ldots \ldots . .$. & 1.56 & 3.23 \\
\hline $13431.121 \ldots \ldots \ldots \ldots \ldots \ldots \ldots \ldots \ldots . .$. & -74.60 & 3.16 \\
\hline 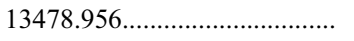 & -13.31 & 4.29 \\
\hline 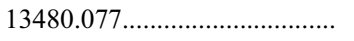 & -70.90 & 4.49 \\
\hline $13480.932 \ldots \ldots \ldots \ldots \ldots \ldots \ldots . .$. & 113.06 & 4.79 \\
\hline $13483.928 \ldots \ldots \ldots \ldots \ldots \ldots$ & -96.96 & 4.90 \\
\hline
\end{tabular}

broadening and to provide the wavelength scale (Butler et al. 1996). Observation dates, RVs, and instrumental uncertainties in the RVs are listed in Table 2. The first three RVs showed an rms scatter of $88 \mathrm{~m} \mathrm{~s}^{-1}$, flagging this star as a planet-bearing candidate. Additional Doppler observations confirmed Keplerian $\mathrm{RV}$ variations consistent with a short-period planet. Based on the chromospheric activity of the star, we expect astrophysical noise in the RVs, or stellar jitter, of about $3.5 \mathrm{~m} \mathrm{~s}^{-1}$ (Wright 2005). For purposes of fitting a Keplerian model, this stellar jitter was added in quadrature to the formal instrumental errors. The best-fit Keplerian model yields an orbital period $P=4.072 \pm 0.7$ days, eccentricity $e=0.016 \pm 0.01$, and a velocity semiamplitude $K$ $=149.6 \pm 3.0 \mathrm{~km} \mathrm{~s}^{-1}$. The rms to the fit is $4.72 \mathrm{~m} \mathrm{~s}^{-1}$, with $\left(\chi_{\nu}^{2}\right)^{1 / 2}=1.11$. Orbital parameters for this fit are summarized in Table 3. Uncertainties in the orbital parameters were determined by running 100 Monte Carlo trials. In each trial, the theoretical fit was subtracted and the residual velocities were scrambled before adding the theoretical velocities back in and obtaining a new fit. From the best-fit orbital elements and the adopted stellar mass of $1.21 M_{\odot}$, we derive $M \sin i=1.33 M_{\mathrm{J}}$ and an orbital radius of $0.053 \mathrm{AU}$. The Keplerian orbital solution is listed in Table 3 and the phased RV data is plotted with the Keplerian model (solid line) in Figure 2.

TABLE 3

Orbital Parameters for HD 149143b and HD 109749b

\begin{tabular}{|c|c|c|}
\hline Parameter & HD 149143b & HD 109749b \\
\hline$P$ (days) & $4.072(0.70)$ & $5.240(0.002)$ \\
\hline$T_{p}(\mathrm{JD}) \ldots \ldots \ldots \ldots \ldots \ldots$ & $2453483.9(1.2)$ & $2453013.55(0.14)$ \\
\hline$\omega(\mathrm{deg})$ & 0 (fixed) & 0.0 (fixed) \\
\hline Eccentricity ..................... & $0.016(0.01)$ & $0.01(0.01)$ \\
\hline$K_{1}\left(\mathrm{~m} \mathrm{~s}^{-1}\right) \ldots \ldots \ldots \ldots$ & $149.6(3.0)$ & $28.3(0.6)$ \\
\hline$a(\mathrm{AU})$ & 0.053 & 0.0635 \\
\hline$a_{1} \sin i(\mathrm{AU}) \ldots \ldots \ldots \ldots$ & $5.57 \mathrm{E}-05$ & $1.38 \mathrm{E}-05$ \\
\hline$f_{1}(m)\left(M_{\odot}\right) \ldots \ldots \ldots \ldots \ldots$ & $1.39 \mathrm{E}-09$ & $1.287 \mathrm{E}-11$ \\
\hline$M \sin i\left(M_{\mathrm{J}}\right) \ldots \ldots \ldots \ldots \ldots$ & 1.33 & $0.28(0.016)$ \\
\hline$N_{\text {obs }}$ & 17 & 21 \\
\hline $\mathrm{rms}\left(\mathrm{m} \mathrm{s}^{-1}\right) \ldots \ldots \ldots \ldots \ldots$ & 4.72 & 2.77 \\
\hline Reduced $\left(\chi_{\nu}^{2}\right)^{1 / 2} \ldots \ldots \ldots$ & 1.11 & 1.05 \\
\hline
\end{tabular}

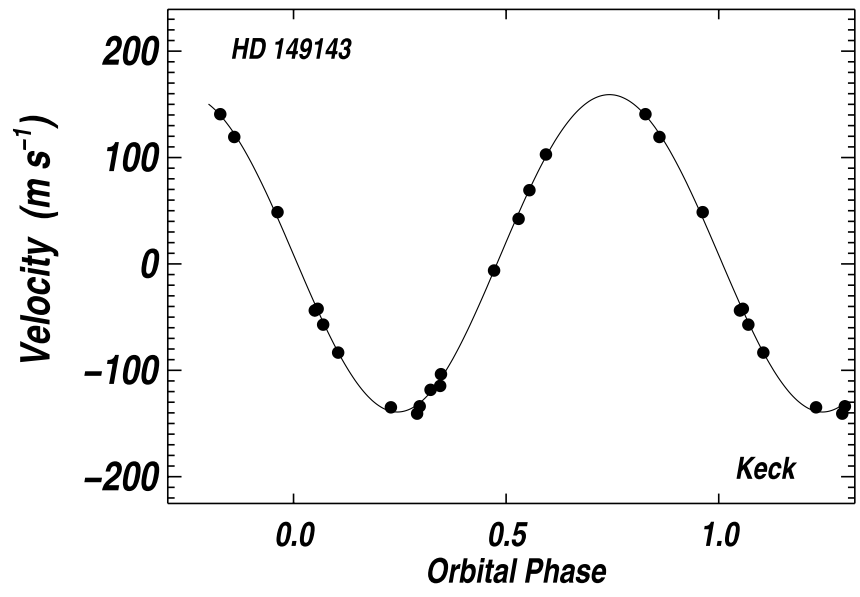

Fig. 2.-Phased RVs for HD 149143 plotted with a Keplerian model (solid line) for a planet with an orbital period of $4.072 \pm 0.7$ days, $M \sin i=1.33 M_{\mathrm{J}}$, and circular orbit with an orbital radius of $0.053 \mathrm{AU}$.

\subsection{Photometric Observations of HD 149143}

We observed HD 149143 with the T12 $0.8 \mathrm{~m}$ automatic photometric telescope (APT) at Fairborn Observatory between 2005 February and July to look for low-amplitude photometric variations in the star and to search for transits of the planetary companion. Queloz et al. (2001) and Paulson et al. (2004) have shown that photospheric features such as spots and plages on solar-type stars can result in low-amplitude, periodic RV variations capable of mimicking the presence of a planetary companion. The rare transiting systems allow the determination of the true mass, radius, and mean density of the planet (e.g., Paper II). Therefore, photometric observations are a useful complement to the Doppler velocity observations.

The T12 APT is equipped with a two-channel precision photometer employing two EMI 9124QB bi-alkali photomultiplier tubes to make simultaneous measurements in the Strömgren $b$ and $y$ passbands. The APT measures the difference in brightness between a program star and a nearby constant comparison star or stars, with a typical precision of 0.0015 mag for bright stars $(V<8.0)$ (Henry 1999). For HD 149143 , we used comparison stars HD $148711(V=6.90, B-V=0.43, \mathrm{~F} 5), \mathrm{HD} 150557$ $(V=5.74, B-V=0.34$, F2.7 III-IV $)$, and HD $150618(V=$ $7.59, B-V=0.40, \mathrm{~F} 2)$. HD 150557 appears to be slightly variable, with an amplitude of $0.001 \mathrm{mag}$ or so, and preliminary period analysis suggests that it may be a $\delta$ Scuti star. Since both HD 148711 and HD 150618 appear to be constant, we have used the average brightness of these two stars as our comparison for HD 149143 to improve our precision. We reduced our Strömgren $b$ and $y$ differential magnitudes with nightly extinction coefficients and transformed them to the Strömgren system with yearly mean transformation coefficients. To improve our photometric precision further, we have averaged the $b$ and $y$ differential magnitudes into a single $(b+y) / 2$ passband. Additional information on the telescope, photometer, observing procedures, and data reduction techniques employed with the T12 APT can be found in Henry (1999) and Eaton et al. (2003).

The resulting $472(b+y) / 2$ differential magnitudes of HD 149143 are given in Table 4 and plotted against Julian date in the top panel of Figure 3. The standard deviation of the observations from the mean brightness level is $0.0022 \mathrm{mag}$, slightly larger than the typical $0.0015 \mathrm{mag}$ precision obtained with the T12 APT. One reason for the larger standard deviation may be 
TABLE 4

Photometric Observations of HD 149143

\begin{tabular}{|c|c|}
\hline HJD $-2,400,000$ & $\begin{array}{c}\Delta(b+y) / 2 \\
(\mathrm{mag})\end{array}$ \\
\hline $53,426.9492 \ldots$ & 0.7157 \\
\hline 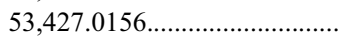 & 0.7169 \\
\hline $53,427.9688 \ldots \ldots \ldots \ldots \ldots \ldots \ldots \ldots \ldots$ & 0.7176 \\
\hline 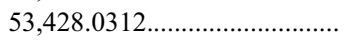 & 0.7186 \\
\hline 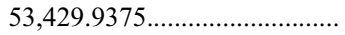 & 0.7177 \\
\hline
\end{tabular}

Note.-Table 4 is published in its entirety in the electronic edition of the Astrophysical Journal. A portion is shown here for guidance regarding its form and content.

HD 149143 's location just $2^{\circ}$ north of the celestial equator, resulting in somewhat higher than average air mass for the observations. However, the top panel of Figure 3 suggests intrinsic night-to-night changes in the brightness of HD 149143 of around $0.002 \mathrm{mag}$, similar in amplitude to observed solar brightness variability (e.g., Willson 1997). The chromospheric activity level of HD 149143 ( Table 1) is also similar to the Sun's (Wright et al. 2004). Periodogram analysis of our photometry reveals a possible weak periodicity around 35 days, comparable to the 28 day rotation period predicted above from the star's chromospheric activity level.

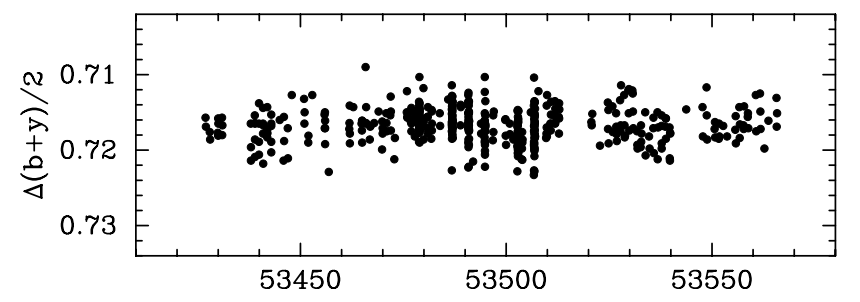

Julian Date - 2,400,000
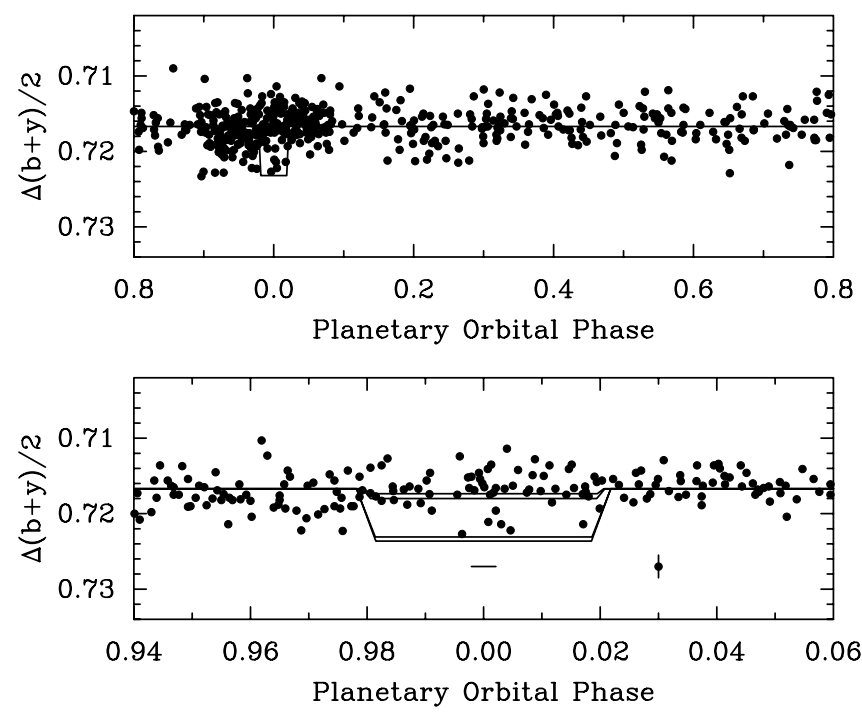

FIG. 3.-Strömgren $(b+y) / 2$ photometric observations of HD 149143 acquired with the T12 $0.8 \mathrm{~m}$ APT at Fairborn Observatory (top) suggesting lowlevel brightness variability of around $0.002 \mathrm{mag}$. The photometric observations are phased to the RV period (middle). The star is constant on the RV period to a limit of $0.0003 \mathrm{mag}$, providing strong support for the planetary interpretation of the RV variations. Predicted transit light curves for a variety of planetary compositions are compared to the observations (bottom). Transits are ruled out for gas giant planets with and without a massive core and are unlikely for denser planets as well.
The 472 photometric observations are replotted in the middle panel of Figure 3, phased with the 4.072 day RV period and an epoch of inferior conjunction (HJD 2453490.8644) computed from the orbital elements in Table 3. A least-squares sine fit of these observations gives a semiamplitude of $0.0003 \pm 0.0002$ mag. This very low limit to possible photometric variability on the RV period supports planetary-reflex motion as the cause of the RV variations.

The photometric observations near phase 0.0 are replotted with an expanded abscissa in the bottom panel of Figure 3 . The solid curves approximate predicted transit light curves for a variety of planetary compositions, assuming a planetary orbital inclination of $90^{\circ}$ (central transits). From deep to shallow, the predicted transit depths correspond to models of an $\mathrm{H} / \mathrm{He}$ gas giant with no core $(0.64 \%)$, an $\mathrm{H} / \mathrm{He}$ gas giant with a $40 M_{\oplus}$ core $(0.59 \%)$, a pure $\mathrm{H}_{2} 0$ planet $(0.12 \%)$, and a pure olivine planet $(0.06 \%)$. The first two models come from Bodenheimer et al. (2003); the last two models are from Guillot (2005). The horizontal bar below the predicted transit window in the bottom panel represents the approximate uncertainty in the time of mid transit. The vertical error bar to the right of the transit window corresponds to the \pm 0.0015 mag measurement uncertainties for a single observation. The geometric probability of transits is $14.7 \%$, computed from equation (1) of Seagroves et al. (2003) assuming random orbital inclinations. The mean of the $67 \mathrm{ob}-$ servations inside the transit window is $0.7166 \pm 0.0003 \mathrm{mag}$; the mean of the remaining 405 observations outside the window is $0.7168 \pm 0.0001 \mathrm{mag}$. Therefore, transit depths greater than $0.02 \%$ or so are ruled out by the observations. This eliminates any possibility of a transiting gas giant with or without a massive core, and makes transits of denser planets unlikely as well. The planetary orbital inclination must be less than $83^{\circ}$.

\section{HD 109749}

HD 109749 is a G3 IV star with $V=8.09$ and $B-V=$ 0.714. The Hipparcos parallax (ESA 1997) of 16.94 mas sets a distance of $59 \mathrm{pc}$, absolute visual magnitude of 4.22, and bolometric luminosity of $1.66 L_{\odot}$. The metallicity estimate from broadband calibration (Ammons et al. 2006) is $0.4 \pm 0.15$ and our high-resolution spectroscopic analysis yields $T_{\text {eff }}=5903 \pm$ $50 \mathrm{~K},[\mathrm{Fe} / \mathrm{H}]=0.25 \pm 0.05, \log \mathrm{g}=4.403 \pm 0.07$, and $v \sin i=$ $2.5 \pm 0.5 \mathrm{~km} \mathrm{~s}^{-1}$. Based on the temperature and luminosity, we derive a stellar radius of $1.24 R_{\odot}$. Interpolation of Yale isochrones provides a stellar mass estimate of $1.2 M_{\odot}$ and age of $3.7 \pm 1.3$ Gyr. HD 109749 is chromospherically inactive, with $S_{\mathrm{HK}}=0.155$ and $\log R_{\mathrm{HK}}^{\prime}=-5.04$. The $\mathrm{Ca} \mathrm{H}$ line is shown in Figure 1. Based on the calibration outlined in Noyes et al. (1984) the implied rotational period is 34 days. Stellar parameters for HD 109749 are listed in Table 1.

\subsection{Doppler Observations of HD 109749}

Doppler RVs for HD 109749 (Table 5) were obtained at the Keck Observatory using HIRES (Vogt et al. 1994). The rms scatter from the first three Doppler observations was only $12 \mathrm{~m} \mathrm{~s}^{-1}$; however, a change in RV of $20 \mathrm{~m} \mathrm{~s}^{-1}$ between the first two nights led us to follow up on this star in search of a short-period planet candidate. A stellar jitter of $3.3 \mathrm{~m} \mathrm{~s}^{-1}$ (Wright 2005) was added in quadrature with the instrumental uncertainties for purposes for fitting the Keplerian model. The best-fit Keplerian model (Fig. 4) yields $P=5.24 \pm 0.01$ days, $K=28.8 \pm 0.6 \mathrm{~m} \mathrm{~s}^{-1}$, and $e=0.01 \pm 0.01$. The rms to this fit is $2.55 \mathrm{~m} \mathrm{~s}^{-1}$ with $\left(\chi_{\nu}^{2}\right)^{1 / 2}=1.05$. The orbital parameters are listed in Table 3 . From these orbital parameters and the stellar mass of $1.2 M_{\odot}$, 
TABLE 5

Radial Velocities for HD 109749

\begin{tabular}{|c|c|c|}
\hline $\mathrm{JD}-2,440,000$ & $\begin{array}{c}\mathrm{RV} \\
\left(\mathrm{m} \mathrm{s}^{-1}\right)\end{array}$ & $\begin{array}{c}\text { Uncertainty } \\
\qquad\left(\mathrm{m} \mathrm{s}^{-1}\right)\end{array}$ \\
\hline $13015.16544 \ldots \ldots \ldots \ldots \ldots$ & -1.73 & 3.92 \\
\hline $13016.15352 \ldots \ldots \ldots \ldots \ldots$ & -23.67 & 4.41 \\
\hline $13017.14260 \ldots \ldots \ldots \ldots \ldots$ & -3.85 & 4.31 \\
\hline $13069.00154 \ldots \ldots \ldots \ldots \ldots$ & -20.28 & 4.09 \\
\hline $13153.77870 \ldots \ldots \ldots \ldots \ldots$ & 2.50 & 3.26 \\
\hline $13425.04097 \ldots \ldots \ldots \ldots \ldots$ & -20.87 & 2.87 \\
\hline $13425.95603 \ldots \ldots \ldots \ldots \ldots$ & -0.93 & 2.38 \\
\hline $13426.99509 \ldots \ldots \ldots \ldots \ldots$ & 28.35 & 2.28 \\
\hline $13428.10716 \ldots \ldots \ldots \ldots \ldots$ & 26.20 & 2.88 \\
\hline $13429.05822 \ldots \ldots \ldots \ldots \ldots$ & -6.17 & 2.89 \\
\hline $13429.96628 \ldots \ldots \ldots \ldots \ldots$ & -20.25 & 2.32 \\
\hline $13431.09335 \ldots \ldots \ldots \ldots \ldots$ & -2.96 & 2.65 \\
\hline $13478.93684 \ldots \ldots \ldots \ldots \ldots$ & 18.18 & 2.68 \\
\hline $13480.84783 \ldots \ldots \ldots \ldots \ldots$ & 19.33 & 2.64 \\
\hline $13483.83481 \ldots \ldots \ldots \ldots \ldots$ & 1.69 & 2.59 \\
\hline $13546.74890 \ldots \ldots \ldots \ldots \ldots$ & 0.78 & 3.07 \\
\hline $13547.76182 \ldots \ldots \ldots \ldots \ldots$ & 31.38 & 2.26 \\
\hline $13548.78374 \ldots \ldots \ldots \ldots \ldots$ & 25.83 & 2.94 \\
\hline $13549.78067 \ldots \ldots \ldots \ldots \ldots$ & -13.66 & 3.10 \\
\hline $13550.80459 \ldots \ldots \ldots \ldots \ldots$ & -26.25 & 2.97 \\
\hline $13551.80551 \ldots \ldots \ldots \ldots \ldots$ & 2.83 & 3.04 \\
\hline
\end{tabular}

we derive a planet mass, $M \sin i=0.28 M_{\mathrm{J}}$, with an orbital radius of $0.0635 \mathrm{AU}$.

\subsection{Photometric Observations of HD 109749 with the SMARTS Telescopes}

We observed HD 109749 in 10 visits spread uniformly throughout the night of 2005 April 9-10 with the $1.3 \mathrm{~m}$ (formerly 2MASS) telescope at Cerro Tololo Inter-American Observatory (CTIO), which is operated by the SMARTS (Small and Moderate Aperture Research Telescope System) consortium. AndiCam was used to observe simultaneous in $V$ and $J$ band, with exposure times of 0.5 and $4.0 \mathrm{~s}$ respectively. The $0.5 \mathrm{~s} V$-band exposures are well exposed, and they are illustrated in Figure 5. We made three observations per visit, for a total of 30 observations. We used SAO $223557, \sim 8^{\prime \prime}$ from the target star, to determine the night's extinction coefficient, 0.13 mag per air mass. The standard de-

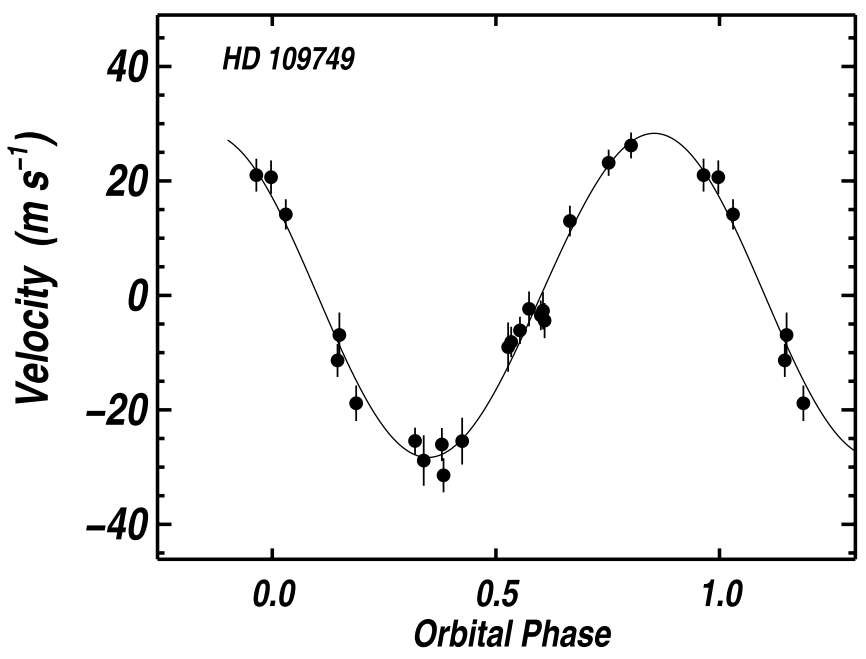

FIG. 4.-Phased RV for HD 109749. With an orbital period of 5.24 days, velocity amplitude of $28.7 \mathrm{~m} \mathrm{~s}^{-1}$, and stellar mass of $1.2 M_{\odot}$, we derive a planet mass $M \sin i=0.28 M_{\mathrm{J}}$ and orbital radius of $0.0635 \mathrm{AU}$.

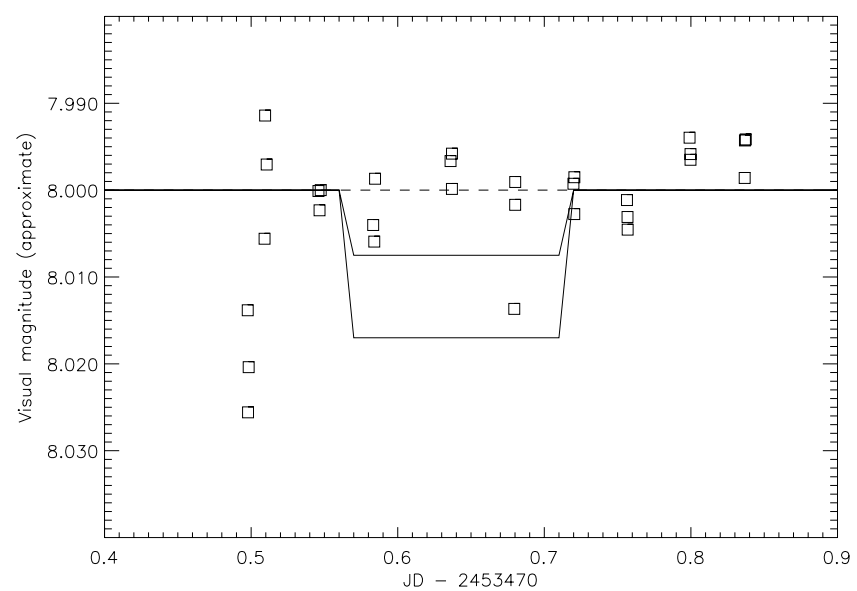

FIG. 5.-Simplified models of two transits and no transit compared to the SMARTS observations of HD 109749. The two models of transits are both centered at $\mathrm{JD}=2,453,470.6448$, have a duration of 0.15 days, and have a depth commensurate with (1) a planet similar in radius to HD 209458b, $r=1.4 r_{\mathrm{J}}$, creating a central obscuration of $0.017 \mathrm{mag}$ and (2) a planet with a central obscuration of $0.0075 \mathrm{mag}$.

viation of all 30 measurements of the target star is $7.7 \mathrm{mmag}$. The scatter within each triplet of observations made in quick succession increases with air mass $A$ approximately as expected for scintillation, $2.8^{1.75} \mathrm{mmag}$ rms per measurement (Dravins et al. 1998; eq.[10]). Shot noise contributes $1.4 \mathrm{mmag}$ rms per measurement. The standard deviation of the 18 observations made at small air mass $(1.02<A<1.27)$ is $4.0 \mathrm{mmag}$. If we assume that the nine observations during the expected transit time are independent and have errors of $4.0 \mathrm{mmag}$ each, then $\chi^{2}=17,30$, and 143 for three possible models: no transit, a transit of $0.7 \%$ depth, and one of $1.7 \%$ depth. The associated probabilities that $\chi^{2}$ for 9 degrees of freedom (dof) is at least that large by chance alone are $0.06,0.0004$, and 0.0000 . That all three probabilities are so small indicates that the observations are not independent. We presume they are correlated on timescales of minutes, due to uncalibrated variations in atmospheric transmission. We average the observations in each triplet within the transit period; the averages differ from the median of all 30 observations by $2.9,-2.6$, and $4.8 \mathrm{mmag}$. If the error of the mean of each triplet is $4.0 \mathrm{mmag}$, then $\chi^{2}=2.4,7.1$, and 45.7 for the three models, respectively, with corresponding $\chi^{2}$ probabilities for 3 dof of $0.49,0.07$, and 0.000 , respectively. Consequently, we state with $93 \%$ confidence that no transit of depth $\gtrsim 0.7 \%$ occurs at the anticipated time.

\subsection{Photometric Observations of HD 109749 with the PROMPT Telescope}

We monitored HD 109749 photometrically on the night of 2005 April 30 to search for transits of the planet across the disk of the star. We gathered images with one telescope of the PROMPT (Panchromatic Robotic Optical Monitoring and Polarimetry Telescopes; Reichart et al. 2006) array, ${ }^{15}$ located at the CTIO. When completed in late 2005, PROMPT will consist of six $0.41 \mathrm{~m}$ Ritchey-Chretien telescopes on rapidly slewing mounts. For the observations presented here, we used a temporary commercially available $0.36 \mathrm{~m}$ Schmidt-Cassegrain telescope. We repeatedly

\footnotetext{
15 The primary goal of the facility will be rapid and simultaneous multiwavelength observations of gamma-ray burst afterglows. In addition to measuring redshifts by dropout and spectral-flux distributions and extinction curves, PROMPT will facilitate quick response observations at $8.1 \mathrm{~m} \mathrm{Gemini}$ South and $4.1 \mathrm{~m}$ SOAR. PROMPT is described in more detail at http:// www.physics.unc.edu/ reichart/prompt.html.
} 
imaged a $12^{\prime}$ square field of view surrounding the target, gathering $R$ images with an integration time of either 1 or $3 \mathrm{~s}$ and a cadence of $30 \mathrm{~s}$. We gathered images continuously from UT 2005 April 430 23:25 until UT 2005 May 5-01 08:42, with a single 8 minute interruption while the German-equatorial mount reacquired the target after it crossed the meridian. We converted the time stamps in the image headers to HJD at mid-exposure. We performed bias subtraction and flat fielding and estimated the centroids of the stellar positions within each image. We evaluated the stellar fluxes within a circular aperture with a diameter of 5.0 pixels (3."5). We produced a relative time series by taking the ratio of the measured fluxes for HD 109749 to those of a close neighbor SAO 223557 $\left(8^{\prime \prime}\right.$ distant) and subsequently renormalized this time series by its median value. Although other bright stars were present in the field of view, we found that position-dependent and time-dependent variations in the point-spread function precluded their use as calibrators. We assigned uncertainties to the normalized time series by adding in quadrature the contributions from scintillation (Dravins et al. 1998, eq. [10]), photon noise, and a constant floor value of $0.75 \%$ that was selected so that the median calculated uncertainty matched the rms variation of $1.7 \%$. We truncated the data set at an air mass of 2, because the photometric precision degraded rapidly with increasing air mass. These data are presented in Table 6 . We then binned the normalized time series by a factor of 10 to obtain an rms variation of $0.57 \%$, very close to the calculated median precision of $0.54 \%$ per binned data point. Both the unbinned and binned time series are shown in Figure 6.

Our time series spans the allowed range of the time of center of transit $T_{c}=2453491.695 \pm 0.030 \mathrm{HJD}$, as predicted from the RV measurements. We see no evidence for any statistically significant variability in the stellar flux. Our models predict a planetary radius of $0.92 R_{\mathrm{J}}$ and $1.09 R_{\mathrm{J}}$ for a planet with and without a substantial core, respectively. Assuming an inclination of $88^{\circ}$ (intermediate within the range of transiting configurations), the corresponding increases in the $\chi^{2}$ for the resulting transit curves are 50.3 and 95.2, respectively. Therefore, we can confidently rule out transits by such an object. Furthermore, we can also exclude $\left(\Delta \chi^{2}=20.5\right)$ transits for a smaller, coredominated planet with a radius of $0.72 R_{\mathrm{J}}$, as was recently deduced for HD 149026b (Paper II). The theoretical transit curves for these three cases are overplotted on the binned data in Figure 6. Taking into account the uncertainty in $T_{c}$, as well as the range of possible inclinations (other than a grazing eclipse), we find that transits remain excluded with a high degree of confidence.

\subsection{Photometric Observations of HD 109749 by Transitsearch.org Collaborators}

Transitsearch.org is a worldwide network of photometric observers operating small independent telescopes. The primary

TABLE 6

PROMPT $R$ PHOTOMETRY OF HD 109749

\begin{tabular}{|c|c|c|}
\hline HJD & Relative Flux & Uncertainty \\
\hline $2,453,491.480670 \ldots$. & 1.0033 & 0.0169 \\
\hline 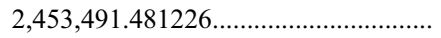 & 0.9870 & 0.0168 \\
\hline 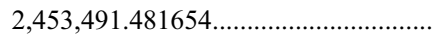 & 0.9915 & 0.0169 \\
\hline 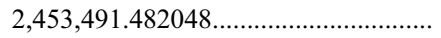 & 0.9977 & 0.0168 \\
\hline 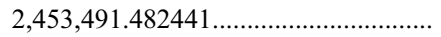 & 0.9888 & 0.0168 \\
\hline
\end{tabular}

NotE.-Table 6 is published in its entirety in the electronic edition of the Astrophysical Journal. A portion is shown here for guidance regarding its form and content. The data are also available in digital format from D. Charbonneau upon request.
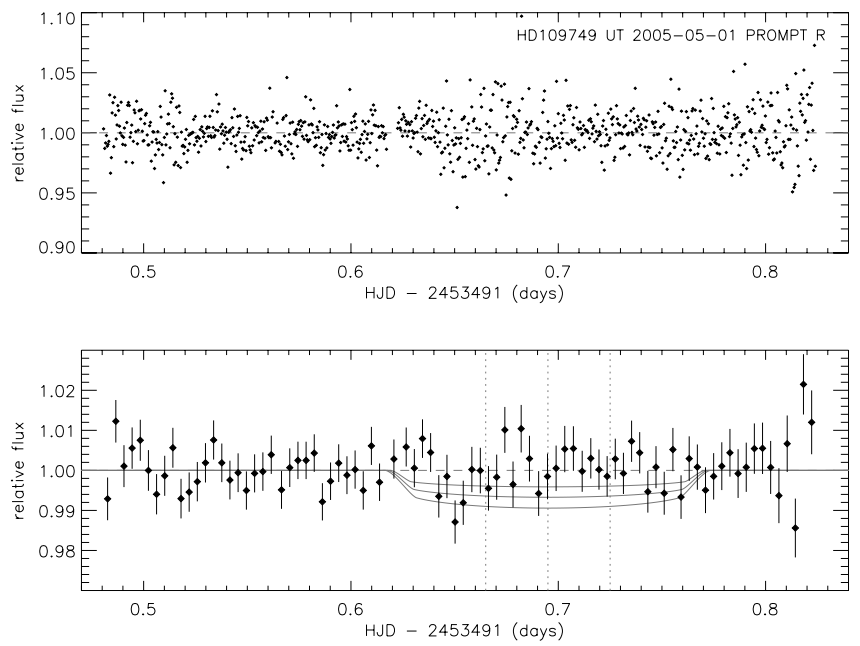

FIG. 6.-Top: PROMPT $R$-band measurements of the relative flux of HD 109749 on the night of 2005 April 30, as described in the text. Bottom: Same flux measurements binned by a factor of 10 , achieving an rms variation of $0.56 \%$. The predicted time of transit center is indicated by the center vertical line, and the uncertainty in this time is indicated by vertical lines to the left and right of the central line. Theoretical transit curves for a planet radius of 1.09 , 0.92 , and $0.72 R_{\mathrm{J}}$ (from most deep to most shallow) are also shown. Based on these data, we may confidently exclude such transits.

goal of the collaboration is to follow up known intermediateperiod (10 days $<P<200$ days) planets to determine whether transits are occurring. Transitsearch.org observers are also providing photometric follow-up observations for N2K candidate short-period planets lying south of decl. $=30^{\circ}$.

On 2005 April 20 we obtained differential aperture photometry of HD 109749 with a Celestron $\mathrm{C} 14$ at $\mathrm{f} / 7$ attached to an SBIG ST-6 CCD. HD 109661 (G1 V, $V=10.26$ ) was used as a comparison star. The observations were made from the University of Adelaide Observatory, Adelaide, Australia. V-filter exposures of $20 \mathrm{~s}$ duration were made at a $60 \mathrm{~s}$ cadence. Six total runs, each of 60 exposures, were accumulated during the interval from HJD 2,453,480.976 through 2,453,481.243. Our best-fit ephemeris yields a predicted central transit epoch $T_{c}=2,453,481.216$

Results from the observations are plotted in the top panel of Figure 7 and are compared with a model light curve based on a 230 minute central transit of HD 109749 by a $1 R_{\mathrm{J}}$ planet and a assuming a linear limb-darkening coefficient, $u=0.6$. Although several small systematic trends can be identified in the data, the time series shows no evidence of a transit at the predicted ingress time.

During the next predicted transit opportunity, which occurred at $T_{c}=$ HJD 2,453,486.46 \pm 0.14 (2005 April 25), HD 109749 was observed at the CBA Pretoria observatory in Pretoria, South Africa. Aperture differential photometry was obtained from HJD 2,453,486.217 through HJD 2,453,486.458, using a $30 \mathrm{~cm}$ SCT telescope equipped with $V$ filter. HD 109661 was again used as the primary comparison star. Individual images were made with $13 \mathrm{~s}$ exposures, with an acquisition rate of four images per minute. Master images were produced by coadding groups of 50 images, yielding a photometric cadence of 13 minutes per light curve point. The resulting time series is shown in the bottom panel of Figure 7.

Imperfections of the alignment and the tracking of the telescope made it necessary to make a position correction at UT 19:10. This repositioning, with attendant imperfect flat fielding around the new position of HD 109749, is likely responsible for sudden shift in the photometric baseline at HJD 2,453,486.3. At 

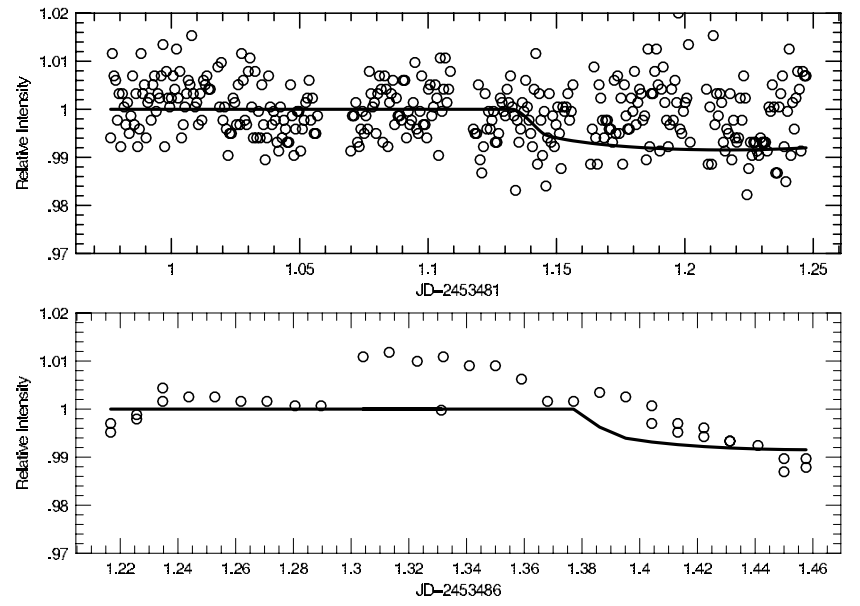

FIG. 7.-Top: $V$-band photometric observations of HD 109749 taken from Adelaide Australia on the night of 2005 April 20. The predicted light curve of a central transit is shown as a solid line. No evidence for a transit is seen. Bottom: V-band photometric observations of HD 109749 taken from Pretoria, South Africa, on the night of 2005 April 25. The predicted light curve of a central transit is shown as a solid line. As explained in the text, the photometric decline in the second half of the data is attributed to a systematic trend and can be discounted. No evidence for a transit is seen.

several times during the observation run, image stacking was executed in manual mode by rejecting images that were subjectively judged to be of inferior quality. The results of the manual and automated stacking are shown as pairs of points sharing the same time stamp. The photometric differences between the two stacking modes were always less than 0.005 mag. The observed decrease in relative intensity during the latter half of the run is roughly in line with the expected transit dip. It was observed, however, that this decline also occurred for a "check" star, pointing to a systematic cause for the decline. Taken together, we conclude that the Adelaide and Pretoria photometry shows no evidence for the occurrence of a transit at the predicted time.

\section{DISCUSSION}

Here, we present the detection of two short-period planets. The first planet orbits the G0 IV star HD 149143, with a period of 4.072 days and planet mass $M \sin i=1.33 M_{\mathrm{J}}$. The second planet was detected around the G3 IV star HD 109749 and has an orbital period of 5.24 days and planet mass $M \sin i=0.28 M_{\mathrm{J}}$.
Both stars were observed as part of the N2K consortium and have high metallicity as part of the sample selection for that program.

Because short-period planets are statistically more likely to be seen in transit, photometric follow-up was initiated. Photometry of HD 149143 was obtained at Fairborn Observatory and demonstrates photometric stability of the host star. Observations made at the Doppler-predicted ephemeris times rule out transit depths down to $0.02 \%$ and constrain the orbital inclination for a gas giant planet to be less than $83^{\circ}$.

HD 109749 is a southern hemisphere object, so photometry was obtained with the SMARTS consortium telescopes, a telescope in the PROMPT array, and by transitsearch.org observers in Adelaide, Australia, and Pretoria, South Africa. Although gas giant planets with a range of chemical compositions could have been observed during a transit, the stellar brightness was constant over the predicted ephemeris times.

We gratefully acknowledge the dedication and support of the Keck Observatory staff, in particular Grant Hill for support with HIRES. We thank Rebeccah Winnick (at Yale University) for scheduling the SMARTS observations. We thank the NOAO and NASA telescope assignment committees for generous allocations of telescope time. Data presented herein were obtained at the W. M. Keck Observatory from telescope time allocated to NASA through the agency's scientific partnership with the California Institute of Technology and the University of California. The Observatory was made possible by the generous financial support of the W. M. Keck Foundation. We thank the Michaelson Science Center for travel support and support through the KDPA program. D. A. F. is a Cottrell Science Scholar of Research Corporation. We acknowledge support from NASA grant NNG05G164G (to D. A. F.); NASA grant NCC5-511 and NSF grant HRD-9706268 (to G. W. H.); NASA grant NAG5-75005 (to G. W. M.); NSF grant AST 99-88358 and NASA grant NAG5-4445 (to S. S. V.); NASA grant NAG5-13285 to P. B.; and NASA grant NNA04CC99A (to G. L.). D. M. is supported by FONDAP N. 15010003. D. E. R. gratefully acknowledges support from NSF's MRI, CAREER, PREST, and REU programs, NASA's APRA, Swift GI, and IDEAS programs, and especially Leonard Goodman and Henry Cox. Work by H. A. K. was supported by a National Science Foundation Graduate Research Fellowship. This research has made use of the Simbad database, operated at CDS, Strasbourg, France.
Alonso, R., et al. 2006, ApJ, in press

Ammons, M., et al. 2006, ApJ, in press

Bodenheimer, P., Laughlin, G., \& Lin, D. N. C. 2003, ApJ, 592, 555

Bouchy, F., Pont, F., Santos, N. C., Melo, C., Mayor, M., Queloz, D., \& Udry, S. 2004, A\&A, 421, L13

Burrows, A., Hubeny, I., \& Sudarsky, D. 2005, ApJ, 625, L135

Butler, R. P., Marcy, G. W., Williams, E., McCarthy, C., Dosanjh, P., \& Vogt, S. S. 1996 , PASP, 108,500

Charbonneau, D., Brown, T. M., Latham, D. W., \& Mayor, M. 2000, ApJ, 529, L45

Charbonneau, D., Brown, T. M., Noyes, R. W., \& Gilliland, R. L. 2002, ApJ 568,377

Charbonneau, D., et al. 2005, ApJ, 626, 523

DeMarque, P., Woo, J-H., Kim, Y-C., \& Yi, S. K. 2004, ApJS, 155, 667

Dravins, D., Lindegren, L., Mezey, E., \& Young, A. T. 1998, PASP, 110, 1118

Eaton, J. A., Henry, G. W., \& Fekel, F. C. 2003, in The Future of Small Telescopes in the New Millennium, Vol. 2, ed. T. D. Oswalt (Dordrecht: Kluwer), 189

ESA. 1997, The Hipparcos and Tycho Catalogs (ESA-SP 1200; Noordwijk: ESA)

\section{REFERENCES}

Fischer, D. A., Marcy, G. W., Butler, R. P., Vogt, S. S., Frink, S., \& Apps, K. 2001, ApJ, 551, 1107

Fischer, D. A., \& Valenti, J. A. 2005, ApJ, 622, 1102

Fischer, D. A., et al. 2005, ApJ, 620, 481

Ford, E., Lystad, V., \& Rasio, F. A. 2005, Nature, 434, 873

Fortney, J. J., Marley, M. S., Lodders, K., Saumon, D., \& Freedman, R. 2005, ApJ, 627, L69

Guillot, T. 2005, Ann. Rev. Earth Planet. Sci., 33, 493

Henry, G. W. 1999, PASP, 111, 845

Henry, G. W., Marcy, G. W., Butler, R. P., \& Vogt, S. S. 2000, ApJ, 529, L41 Kley, W., Lee, M. H., Murray, N., \& Peale, S. J. 2005, A\&A, 437, 727

Konacki, M., Torres, G., Sasselov, D. D., \& Jha, S. 2003, ApJ, 597, 1076

Laughlin, G., \& Chambers, J. 2001, ApJ, 551, L109

Lee, M. H. 2004, ApJ, 611, 517

Lee, M. H., \& Peale, S. 2003, ApJ, 592, 1201

Marcy, G. W., Butler, R. P., Fischer, D. A., Vogt, S. S., Wright, J. T., Tinney,

C. G., \& Jones, H. R. A. 2005, Prog. Theor. Phys. Suppl., 158, 24

Mayor, M., \& Queloz, D. 1995, Nature, 378, 355

Noyes, R. W., Hartmann, L., Baliunas, S. L., Duncan, D. K., \& Vaughan, A. H. 1984, ApJ, 279, 763 
Paulson, D. B., Saar, S. H., Cochran, W. D., \& Henry, G. W. 2004, AJ, 127, 1644

Queloz, D., et al. 2001, A\&A, 379, 279

Reichart, D., et al. 2006, Gamma Ray Bursts in the Afterglow Era, ed. L. Piro, L. Amati, S. Covino, \& B. Gendre, Nuovo Cimento, in press (astro-ph/ 0502429)

Rivera, E. J., \& Lissauer, J. J. 2001, ApJ, 558, 392

Rivera, E., et al. 2005, ApJ, 634, 625

Robinson, S. E., et al. 2006, ApJ, in press

Sato, B., et al. 2005, ApJ, 633, 465 (Paper II)

Seagroves, S., Harker, J., Laughlin, G., Lacy, J., \& Castellano, T. 2003, PASP, 115,1355
Udalski, A. Pietrzyński, G., Szymńaski, M., Kubiak, M., Żebruń, K., Soszyński, I., Szewczyk, O., \& Wyrzykowski, L. 2003, Acta Astron., 53, 133

Udalski, A., Żebruń, K, Szymański, M., Kubiak, M., Soszyński, I., Szewczyk, O., Wyrzykowski, L., \& Pietrzyński, G. 2002a, Acta Astron., 52, 115

Udalski, A., et al. 2002b, Acta Astron., 52, 1

Valenti, J. A., \& Fischer, D. A. 2005, ApJS, 159, 141

Vidal-Madjar, A., Désert, J.-M., Lecavelier des Etangs, A., Hébrard, G., Ballester G. E., Ehrenreich, D., \& Ferlet, R. 2004, ApJ, 604, L69

Vogt, S. S., et al. 1994, Proc. SPIE, 2198, 362

Willson, R. C. 1997, Science, 277, 1963

Wright, J. T. 2005, PASP, 117, 657

Wright, J. T., Marcy, G. W., Butler, R. P., \& Vogt, S. S. 2004, ApJS, 152, 261 